\title{
The pathological and clinical features of anterior lesions of prostate cancer: Evaluation in a single cohort of patients
} \author{
Angelo Porreca ${ }^{1}$ \\ ${ }^{1}$ Department of Urology, Abano Terme Hospital, Abano Terme (PD), Italy; \\ 2 Department of Urology, Università "la Sapienza", Roma, Italy; \\ ${ }^{3}$ European Institute of Oncology, Milan, Italy; \\ ${ }^{4}$ Department of Urology, University of Verona, Italy; \\ ${ }^{5}$ Department of Urology, University of Bologna, Italy
}

Daniele D'Agostino ${ }^{1}$, Paolo Corsi ${ }^{1}$, Michele Colicchia ${ }^{1}$, Daniele Romagnoli ${ }^{1}$, Gian Maria Busetto ${ }^{2}$, Matteo Ferro ${ }^{3}$, Alessandro Tafuri ${ }^{4}$, Matteo Cevenini ${ }^{5}$, Federico Mineo Bianchi ${ }^{5}$, Marco Giampaoli ${ }^{5}$,

\begin{abstract}
Summary
Introduction. The aim of our work is to evaluate the principal differences of the pathological features in prostate cancer (PCa) lesions comparing those in the anterior region of the gland (APCa) to those in the posterior zone (PPCa) among patients who underwent to robotic-assisted radical prostatectomy (RP).

Material and methods. A total of 85 consecutive patients (mean age 66; IQR 62-71) with clinically suspected PCa were studied with multiparametric magnetic resonance of prostate before prostate biopsies. The prostate biopsies were RM-guided (60 inbore biopsy (MR-GB) and 25 Fusion-biopsy (FB). A total of 72 cases were eligible for robotic RP. An experienced genitourinary pathologist reviewed the histopathology of the tissue specimens of the patients after RP. The exclusion criteria were as follows: previous hormonotherapy, radiotherapy and chemotherapy for others cancers.
\end{abstract}

Results. Based on the histological diagnosis, after RP, 68 anterior prostate cancer, and 107 posterior lesions were found. We further subcategorized lesions into peripheral and central zones for each the anterior and posterior lesions. The specific distribution of lesions by pathologic stage was: T2 = 74 (42.3\%), T3a= $87(49.7 \%), T 3 b=12(6.9 \%), T 4=2(1.1 \%)$ cases. Tumor volume of posterior neoplasms ranged from 0.04 to $20.35 \mathrm{~cm}^{3}$, with a median of $3.39 \mathrm{~cm}^{3}$. Anterior tumor volume ranged from 0.17 to $15 \mathrm{~cm} 3$, with a median volume of $2.54 \mathrm{~cm}^{3}$ : PPCa were larger than APCa but the difference in size was not significant. The prostate cancer grade group (GG) I was distributed as $16.6 \%$ and $36 \%$ in anterior and posterior lesions cases. GG II and III was $43.8 \%$ and $31.5 \%$ in anterior and posterior cases, respectively. Comparatively, GG IV-V showed $39.6 \%$ and $32.5 \%$ for anterior and posterior lesions respectively $(p<0.001)$. Extraprostatic extention of neoplasm (EPE) was found more frequently in anterior cases (31.4\%) than in in posterior cases (25.1\%), but without significant difference. Lymphovascular invasion was similar in both the groups: $24 \%$ and $28.6 \%$ in anterior and posterior group, respectively. Anterior lesions showed a significantly higher rate of lymph node metastasis $(9.3 \%)$ than posterior lesions $(3.4 \%)(p<0.005)$.

Conclusion. In our study, we have found EPE, often associated with worse prognosis, more frequently (but not significantly) present in anterior lesions among PCa patients. Although posterior lesions are often related to pT3b stage, in our findings, anterior lesions were more often associated with a more aggressive neoplasm with more frequent nodal involvements.

KEY WORDS: Prostate cancer; Anterior lesion; Multiparametric magnetic resonance.

Submitted 7 May 2020; Accepted 13 May 2020

\section{INTRODUCTION}

Prostate cancer ( $\mathrm{PCa}$ ) is the second most frequently diagnosed tumor in males globally (1). Many national and international efforts are ongoing to improve PCa diagnosis, treatment, and, ultimately, the quality of life of patients (2). The majority of tumors have an indolent clinical course, although some cancers have an aggressive and potentially lethal evolution, if they are not promptly treated. PCa is often found in the peripheral area of the prostate gland, although histopathological studies from radical prostatectomy (RP) samples have shown that up to $30 \%$ of clinically significant neoplasms ( $\mathrm{cs} P \mathrm{~Pa})$ can be located in the anterior portion of the gland (APCa), and these are increasing in prevalence $(3,4)$. Cancers that arise in the anterior zone may be difficult to palpate by digital rectal examination (DRE), and are often missed (5). Moreover, transrectal ultrasound (TRUS)-guided prostate biopsy fails to accurately assess the anterior zone of the prostate, where cancers may not be sampled.

Various studies have shown that targeted MRI/ultrasound fusion biopsy (TB), compared with standard biopsy $(\mathrm{SB})$, is associated with increased detection of high-risk prostate cancer and decreased detection of low-risk prostate cancer (6-8).

Many authors $(4,5)$ report that there is no difference in terms of Gleason grade (GG) IV or V rate between patients with APCa or posterior (P)PCa, although APCa has smaller tumor volume and shows a higher rate of positive surgical margins after RP when compared with PPCa $(5,9)$. Additionally, anterior cancers tend to be more aggressive than posterior ones, so early detection of anterior prostate cancer is clinically important (9-11). The aim of the present study is to compare the detection of anterior and posterior PCa in a contemporary cohort of Caucasian patients, admitted to the hospital for suspected PCa diagnosis and to evaluate clinical and pathological features between APCa and PPCa. The investigation was prompted by the finding that, in our practice of RP specimens, tumor volume is lower and GG is higher in a majority of cases, when the index tumor is predominantly located in the anterior region of the gland, compared to the posterior zone. 


\section{MATERIALS AND METHOdS}

A total of 85 consecutive patients (mean age 66; IQR 6271) with clinically suspected PCa were enrolled at our institution between January 2016 and January 2019.

All enrolled patients had been studied with multiparametric magnetic resonance (mpMRI) of prostate before prostate biopsies. We identified 386 suspected lesions at mpMRI (excluding the lesion of transitional zone we included in the evaluation 229 lesions of posterior region and 157 in anterior region of prostate). The demografics and radiological details of cohort are summarized in Table 1. The prostate biopsies were all RM-guided, inbore biopsy (MR-GB) and Fusion-biopsy (FB). According to the risk category, patients were offered active surveillance (AS), robot assisted radical prostatectomy (RARP), or radiation therapy (RT). All details are clarified in Table 2 A total of 72 cases were eligible for robotic RP. The study was a retrospective analysis with the approval by the Ethics Committee Institutional Review Board of Abano Policlinic and signed informed consent was provided by all patients. An experienced genitourinary pathologist reviewed the histopathology of the tissue specimens of the patients after RP. The pathologist, reviewing all prostatectomy tissue sections, identified PCa foci larger than $5 \mathrm{~mm}$ in diameter. The exclusion criteria were as follows: previous hormonotherapy and radiotherapy, chemotherapy

\section{Table 1.}

Overall and stratified according to bioptic status clinical and radiologic features of patients undergoing target biopsy of prostatic anterior lesion identified at mpMRI.

\begin{tabular}{|c|c|c|c|c|}
\hline & $\begin{array}{c}\text { Overall } \\
(n=386)\end{array}$ & $\begin{array}{l}\text { Posterior } \\
\text { lesions } \\
\text { (n= 229) }\end{array}$ & $\begin{array}{c}\text { Anterior } \\
\text { lesions } \\
(n=157)\end{array}$ & p-value \\
\hline \multicolumn{5}{|l|}{ Age } \\
\hline Median & 66 & 67 & 66 & 0.7 \\
\hline IQR & $62-71$ & $61-72$ & $63-71$ & \\
\hline \multicolumn{5}{|l|}{ PSA (ng/ml) } \\
\hline Median & 7 & 6.7 & 7.7 & 0.02 \\
\hline IQR & $5-9.2$ & 4.8-9.1 & $5.2-10.1$ & \\
\hline \multicolumn{5}{|l|}{ Prostate volume (ml) } \\
\hline Median & 53.7 & 55 & 51.2 & 0.5 \\
\hline IQR & $42-69.1$ & $43-69.7$ & $38.1-67.8$ & \\
\hline \multicolumn{5}{|l|}{ PSA density } \\
\hline Median & 0.12 & 0.12 & 0.15 & $<0.001$ \\
\hline$\underline{I Q R}$ & $0.09-0.18$ & $0.09-0.16$ & $0.09-0.23$ & \\
\hline \multicolumn{5}{|l|}{ DRE $(\%)$} \\
\hline Negative & $260(67.4)$ & $161(70.3)$ & $106(67.5)$ & 0.5 \\
\hline Positive & $126(32.6)$ & $77(33.6)$ & $47(29.9)$ & \\
\hline Previous TRUS-GB (\%) & $141(36.6)$ & $69(30.1)$ & $72(45.9)$ & 0.002 \\
\hline \multicolumn{5}{|l|}{ PiRADS score (\%) } \\
\hline 3 & $145(37.6)$ & $105(45.9)$ & $40(25.5)$ & $<0.001$ \\
\hline 4 & $136(35.2)$ & $83(36.2)$ & $53(33.8)$ & \\
\hline 5 & $105(27.2)$ & $41(17.9)$ & $64(40.8)$ & \\
\hline \multicolumn{5}{|c|}{ Index kesion diameter (mm) } \\
\hline Median & 14 & 13 & 16 & $<0.001$ \\
\hline IQR & $11-19$ & $9-17$ & $12-23$ & \\
\hline \multicolumn{5}{|l|}{ Index kesion site (\%) } \\
\hline Peripheral & $277(71.8)$ & $183(79.9)$ & $94(59.9)$ & $<0.001$ \\
\hline Central & $109(28.2)$ & $46(20.1)$ & $63(40.1)$ & \\
\hline
\end{tabular}

Table 2.

Overall and stratified according to Fusion and In-bore biopsy bioptic outcomes of patients undergoing target biopsy of prostatic anterior lesion identified at $\mathrm{mpMRI}$.

\begin{tabular}{|c|c|c|c|c|}
\hline & $\begin{array}{c}\text { Overall } \\
(n=386)\end{array}$ & $\begin{array}{l}\text { Posterior } \\
\text { lesions } \\
(n=229)\end{array}$ & $\begin{array}{c}\text { Anterior } \\
\text { lesions } \\
(n=157)\end{array}$ & p-value \\
\hline \multicolumn{5}{|c|}{ Targeted biopsy technique (\%) } \\
\hline MR-GB & $217(56.2)$ & $118(51.5)$ & $99(63.1)$ & \multirow[t]{2}{*}{0.3} \\
\hline Fusion & $169(43.8)$ & $111(49.5)$ & $58(36.9)$ & \\
\hline \multicolumn{5}{|c|}{ Number of cores taken } \\
\hline Median & 12 & 12 & 12 & \multirow[t]{2}{*}{0.09} \\
\hline IQR & $2-14$ & $2-14$ & $2-14$ & \\
\hline \multicolumn{5}{|l|}{ Positive cores* } \\
\hline Median & 1 & 1 & 2 & \multirow[t]{2}{*}{$<0.001$} \\
\hline IQR & $0-3$ & $0-2$ & $1-3$ & \\
\hline \multicolumn{5}{|l|}{ Gleason grade (\%) } \\
\hline Negative & $138(35.8)$ & $102(44.5)$ & $36(22.9)$ & \multirow{6}{*}{$<0.001$} \\
\hline 1 & $64(16.6)$ & $38(16.6)$ & $26(16.6)$ & \\
\hline 2 & $90(23.3)$ & 37 (16.2) & $53(33.8)$ & \\
\hline 3 & $65(16.8)$ & 35 (15.3) & $30(19.1)$ & \\
\hline 4 & $26(6.7)$ & $15(6.6)$ & $11(7)$ & \\
\hline 5 & $3(0.8)$ & $2(0.9)$ & $1(0.6)$ & \\
\hline \multicolumn{5}{|l|}{ Indication (\%) } \\
\hline No treatment & $138(35.8)$ & $102(44.5)$ & $36(22.9)$ & \multirow{5}{*}{$<0.001$} \\
\hline Active surveillance & $43(11.1)$ & $32(14)$ & $11(7)$ & \\
\hline RP & $175(45.3)$ & $72(31.4)$ & $103(65.6)$ & \\
\hline RT & $23(6)$ & $18(7.9)$ & $5(3.2)$ & \\
\hline ADT & $7(1.8)$ & $5(2.2)$ & $2(1.3)$ & \\
\hline
\end{tabular}

for others cancers. Based on the histological diagnosis, 68 anterior and 107 posterior lesions were found. We also subcategorized the lesions into peripheral and central zones for each anterior and posterior group. The specific distribution of lesions by pathologic stage was: $\mathrm{T} 2=$ $74(42.3 \%), \mathrm{T} 3 \mathrm{a}=87(49.7 \%), \mathrm{T} 3 \mathrm{~b}=12(6.9 \%), \mathrm{T} 4=2$ (1.1\%) cases (ref Table 3).

\section{Pathology protocol}

The radical prostatectomy specimens were fixed in 10\% formalin and cut into approximately $5 \mathrm{~mm}$ sections by hand as follows: apex and base in coronal plane, seminal vesicles in sagittal plane, and mid-gland in transverse plane, perpendicular to the long axis of the urethra.

The 5-mm paraffin-embedded slices blocks were sectioned into 5- $\mu \mathrm{m}$-thick sections and stained with hematoxylin and eosin (H\&E).

Dedicated pathologists examined surgical specimens, which were processed according to the Stanford protocol (30). ISUP grade group system was applied to classify tumors (31). Surgical margins were reported positive when cancer invaded the inked surface of the specimen. Lymph nodes were assessed for histopathology after hematoxylin and eosin staining. Immuno-histochemical staining was performed when appropriate. In each case, the number of removed lymph nodes and LNI was reported. Prostate and nodal specimens were then staged according to the 2010 AJCC staging system for PCa (18).

\section{Perioperative-features}

In each case, clinical pelvic lymph node staging $(\mathrm{cN})$ was 
Table 3.

Overall pathologic outcomes of patients who underwent radical prostatectomy $(n=72)$.

\begin{tabular}{|lc|}
\hline & Overall \\
\hline Pathologic stage (\%) & $74(42.3)$ \\
T3a & $87(49.7)$ \\
T3b & $12(6.9)$ \\
T4 & $2(1.1)$ \\
\hline Pathologic ISUP grade (\%) & $10(5.7)$ \\
1 & $70(40)$ \\
2 & $56(32)$ \\
3 & $34(19.4)$ \\
4 & $5(2.9)$ \\
5 & $113(64.6)$ \\
\hline Pathologic nodal status (\%) & $10(5.7)$ \\
N0 & $52(29.7)$ \\
N1 & $11(6.3)$ \\
Nx & \\
\hline Positive surgical margins (\%) & $68(39.9)$ \\
\hline Pathologic index lesion (\%) & $107(61.1)$ \\
Anterior & \\
Posterior & \\
\hline mpMRI: multiparametric Magnetic Resonance Imaging; ADT: androgen deprivation therapy; \\
RT: radiotherapy; RP: radical prostatectomy; * among those with positive biopsies. \\
\end{tabular}

performed by axial imaging modalities (computed tomography CT or MRI). Enlarged pelvic nodes larger than one centimeter in diameter were staged as $\mathrm{cNl}$ disease. The metastatic status was investigated by both axial imaging and total bone scan modalities. Patients were staged according to 2010 American Joint Committee on Cancer (AJCC) staging system for PCa ( $7^{\text {th }}$ edition) (18). PCa patients were divided into low, intermediate and high risk, according to the D'Amico risk classification (19). In high risk patients in the RARP group, extend pelvic lymph node dissection (ePLND) was performed (20, 21). In intermediate risk patients, the decision to perform an extended lymph node dissection was mainly based on

Table 4.

Uni- and multivariate analysis model predicting features of patients who underwent.

\begin{tabular}{|c|c|c|c|c|}
\hline & \multirow{2}{*}{$\begin{array}{l}\text { Univariate analysis } \\
\text { OR }(95 \% \mathrm{Cl})\end{array}$} & \multicolumn{3}{|c|}{ Multi-variate analysis } \\
\hline & & p-value & OR (95\% Cl) & p-value \\
\hline Age (yrs) & $1.09(1.05-1.12)$ & $<0.001$ & $1.07(1.03-1.12)$ & 0.002 \\
\hline PSA (ng/ml) & $1.12(1.06-1.17)$ & $<0.001$ & $1.15(1.06-1.24)$ & 0.001 \\
\hline Prostate volume (ml) & $0.98(0.97-0.99)$ & $<0.001$ & $0.97(0.95-0.98)$ & $<0.001$ \\
\hline Digito-rectal examination & $5.99(3.69-9.71)$ & $<0.001$ & $7.03(3.76-13.16)$ & $<0.001$ \\
\hline Previous TRUS-GB & $1.55(1.02-2.35)$ & 0.04 & $1.03(0.59-1-8)$ & 0.9 \\
\hline $\mathrm{N}$ of bioptic cores & $1.02(0.99-1.06)$ & 0.3 & - & - \\
\hline \multicolumn{5}{|l|}{ PIRADS score } \\
\hline 3 & Ref. & & Ref. & \\
\hline 4 & $5.63(3.27-9.68)$ & $<0.001$ & $4.08(2.17-7.67)$ & $<0.001$ \\
\hline 5 & $17.27(9.17-32.53)$ & $<0.001$ & $9.6(3.77-24.45)$ & $<0.001$ \\
\hline Index lesion diameter (mm) & n) $1.08(1.05-1.12)$ & $<0.001$ & $0.98(0.92-1.03)$ & 0.7 \\
\hline \multicolumn{5}{|l|}{ mpMRI index lesion location } \\
\hline Posterior & Ref. & $<0.001$ & Ref. & 0.01 \\
\hline Anterior & $2.41(1.59-3.65)$ & & $2.09(1.19-3.68)$ & \\
\hline
\end{tabular}

pre-operative nomograms showing a risk of lymph node invasion greater than 5\% (22). In low risk patients, the decision to perform an ePLND was based on clinical factors indicating increased risk of tumor upgrading and lymph node invasion in the surgical specimen (23).

Skilled and experienced surgeons performed RARP with ePLND using the da Vinci Robot Surgical System (Intuitive Surgical, Inc, Sunnyvale, CA, USA). All procedures were performed through a trans-peritoneal approach with anterograde prostatic dissection (24). Urethro-vesical anastomosis was performed using barbed sutures as previously described (25-26). The lymph node dissection template included bilateral external iliac lymph nodes until the crossing of the ureter and the external iliac artery.

\section{Statistical analysis}

Continuous variables were expressed as median and interquartile range (IQR) whereas categorical variables were expressed as frequencies with percentages. The independent-samples T-test and Chi-Square test were used to compare means and frequencies between the two groups, respectively. All data were statistically analyzed using SPSS v 21 for Macintosh.

\section{Results}

Median age was 66 (IQR 63-71) and 67 (range 61-72) years among anterior and posterior lesions cases, respectively. Mean serum PSA level was 7.7 ng/mL (IQR 5.210.1) in anterior cases and 6.7 $\mathrm{ng} / \mathrm{mL}$ (IQR 4.8-9.1) in posterior cases.

Tumor volume of posterior neoplasms ranged from 0.04 to $20.35 \mathrm{~cm}^{3}$, with a median of $3.39 \mathrm{~cm}^{3}$. Tumor volume of anterior cases ranged from 0.17 to $15 \mathrm{~cm}^{3}$, with a median volume of $2.54 \mathrm{~cm}^{3}$. This difference in size was not statistically significant ( $p>0.05$ ).

The GG I was distributed as $16.6 \%$ and $36 \%$ in anterior and posterior lesions cases, respectively. GGII and III was $43.8 \%$ and $31.5 \%$ in anterior and posterior cases, respectively. GG IV-V was $39.6 \%$ and $32.5 \%$ for anterior and posterior lesions, respectively ( $p<0.001)$.

Extraprostatic extension of neoplasm (EPE) was found more frequently in anterior cases (31.4\%) than in in posterior cases (25.1\%), but without significant difference $(p>0.05)$. Pathologic stages among patients with primary posterior lesions were as follows: $42.3 \%$ in pT2, $49.7 \%$ in pT3a, $6.9 \%$ in pT3b, and $1.1 \%$ in T4. Among patients with anterior primary lesions, in $38.0 \%$ were in pT2 stage, $5.3 \%$ were in pT3a, 5.6\% were in pT3b, and $11.1 \%$ in $\mathrm{T} 4$. Lymphovascular invasion was similar in both the groups: $24 \%$ and $28.6 \%$ in anterior and posterior group respectively. Anterior lesions showed a significantly higher rate of lymph node metastasis (9.3\%) than in posterior lesions $(3.4 \%)(p<0.005)$.

\section{Discussion}

In the literature, the imaging techniques for prostate cancer are in constantly evolving, and several different examination techniques play a fundamental role in the diagnosis, staging (27), and choice of therapeutic approach (28). In particular, the localization of prostate cancer foci with 
mpMRI is crucial in the planning the best diagnostic plane and surgical approach, for example, in robotic surgery (29-31). The pathological and biological features of prostate cancer lesion have been analyzed by numerous studies. The anatomical and biological behavior of APCa are unique, and the definition varies. In the study by Villers and colleagues, they defined the anterior borders of the prostate as the region of parenchyma at least $2.1 \mathrm{~cm}$ anterior to the posterior capsule which is an area that the transrectal biopsy needle characteristically fails to reach (32). Anatomically, this is a portion of the prostate anterior to the urethra which includes areas of McNeal's transition zone, the anterior fibromuscular stroma and the anterior horns of the peripheral zone. These features make the use of MRI-guided prostate biopsy techniques indispensable to obtain a correct therapeutic approach; late identification of anterior lesions significantly affects disease prognosis and surgical outcomes (extra-prostatic extension and positive surgical margins).

Here, we have studied the differences between neoplasms arising from anterior and posterior gland. In retrospective studies of RP, it is reported that over $50 \%$ of tumors are located in anterior prostate (33-34). In agreement with the literature, we found $40 \%$ of lesions were located in anterior gland. At the time of diagnosis, anterior lesions were bigger than posterior ones, because APCa are commonly more advanced and have positive surgical margins (PSM) on RP specimens so therefore they can carry more risk for the patient (35). However, the findings from the present investigation do not support the observed trend from the literature, that anterior lesions have a lover GG than posterior (36-37).

We found that anterior lesions have a higher GG than posterior lesions $(\mathrm{p}<0.005)$ and a more advanced local stage at the time of detection, even in presence of a smaller volume of neoplasm.

Anatomy of the anterior extraprostatic space which spans across the apex through the base, is unique in that the capsule is vaguely defined and is covered with fibromuscular shielding (anterior fibromuscular stroma) AFMS (8). Not only does the lack of capsular structure makes it difficult to define EPE and PSM in these regions, but the particular histological structure may provide an alternative route through which malignant cells can spread and gain access to the lymphatic drainage system. In most studies in literature, APCa are reported to be associated with lower rate of EPE while PSM rate was higher compared to posterior cancers.

Differences in EPE between anterior and posterior lesions result from mechanical/anatomical distinctions, rather than biological differences (38), thought we have found that EPE was slightly more common in anterior lesions, but not statistical so. To better clarify this discrepancy, further anatomopathological studies must be carried out. In accordance with the literature, the present study has found higher rates of seminal vesicles invasion (pT3b) in the posterior lesions than in the anterior lesions, probably correlated to the anatomical location of the seminal vesicle (39). Certainly, an interesting aspect is represented by lymph node diffusion. In our cohort, more lymph nodal involvement has been found in anterior lesions than in posterior ones.
This study has some limitations predominantly related to the retrospective design and the small size of the cohort. Additionally, selection bias may be due to patients who underwent MRI and fusion prostate biopsy were not all patients with a clinical suspect of PCa. Clinicians were used to suggest MRI (+/- biopsy) in more challenging clinical scenario. Moreover, the patients who underwent RP could be a selected subgroup of patients with a longer life expectancy and/or with a more aggressive PCa since lowrisk $\mathrm{PCa}$ might have been managed expectantly with active surveillance. Certainly, a prospective evaluation of pathological and clinical features and long term follow-up of APCa with a major number of cases will be useful to better investigate the real impact of PCa location within the gland.

\section{Conclusions}

Our anatomo-topographic study shows that the anterior prostatic lesions, although smaller than posterior tends to have a higher pathological grade. In contrast with others, we have found EPE, often associated at a worse prognosis, more likely present in anterior lesions. Although, posterior lesions are often related to $\mathrm{pT} 3 \mathrm{~b}$ stage, in our findings, anterior lesions are associated to a more aggressive neoplasm with more frequent nodal involvements.

\section{References}

1. Center MM, Jemal A, Lortet-Tieulent J, et al. International variation in prostate cancer incidence and mortality rates. Eur Urol. 2012; 61:1079-92.

2. Noale M, Maggi S, Artibani W, et al. Pros-IT CNR: an Italian prostate cancer monitoring project. Aging Clin Exp Res. 2017; 29:165-72.

3. Sahu M, Wijesekera N, Donohue JF. Anterior prostate cancer: Current perspectives and diagnostic dilemmas. J Clin Urol. 2017; 10:49-55.

4. Wright JL, Ellis WJ. Improved prostate cancer detection with anterior apical prostate biopsies. Urol Oncol. 2006; 24:492-495.

5. Mygatt J, Sesterhenn I, Rosner I, et al. Anterior tumors of the prostate: clinicopathological features and outcomes. Prostate Cancer Prostatic Dis. 2014; 17:75-80.

6. Kasivisvanathan V, et al. MRI-targeted or standard biopsy for prostate-cancer diagnosis. N Engl J Med. 2018; 378:1767-1777.

7. Siddiqui MM, Rais-Bahrami S, Turkbey B, et al. Comparison of MR/ultrasound fusion-guided biopsy with ultrasound-guided biopsy for the diagnosis of prostate cancer. JAMA. 2015; 313:390-397.

8. Kasivisvanathan V, Rannikko AS, Borghi M, et al. MRI-targeted or standard biopsy for prostate-cancer diagnosis. New Engl J Med. 2018; 378:1767-779.

9. Koppie TM, Bianco Jr FJ, Kuroiwa K, et al. The clinical features of anterior prostate cancers. BJU Int. 2006; 98:1167-71.

10. Lawrentschuk N, Haider MA, Daljeet N, et al. "Prostatic evasive anterior tumours": the role of magnetic resonance imaging. BJU Int. 2010; 105:1231e6.

11. Mai KT, Moazin M, Morash C, et al. Transitional zone and anterior peripheral zone of the prostate. A correlation of small-volume cancer in the biopsy cores and high psa with positive anterior margins in radical prostatectomy specimens. Urol Int. 2001; 66:191e6. 
12. Cerantola Y, Haberer E, Torres J, et al. Accuracy of cognitive MRI-targeted biopsy in hitting prostate cancer-positive regions of interest. World J Urol. 2016; 34:75-82.

13. Gayet M, van der Aa A, Beerlage HP, et al. The value of magnetic resonance imaging and ultrasonography (MRI/US)-fusion biopsy platforms in prostate cancer detection: a systematic review. BJU Int. 2016; 117:392-400.

14. Tan N, Lin W-C, Khoshnoodi P, et al. In-bore 3-T MR-guided transrectal targeted prostate biopsy: Prostate Imaging Reporting and Data System version 2-based diagnostic performance for detection of prostate cancer. Radiology. 2016; 283:130-9.

15. Schiavina R, Vagnoni V, D'Agostino D, et al. "In-bore" MRIguided prostate biopsy using an endorectal nonmagnetic device: a prospective study of 70 consecutive patients. Clin Genitourin Cancer. 2017; 15:417-27.

16. Barentsz JO, Richenberg J, Clements R, et al. ESUR prostate MR guidelines 2012. Eur Radiol. 2012; 22:746-57.

17. Weinreb JC, Barentsz JO, Choyke PL, et al. PI-RADS Prostate Imaging-Reporting and Data System: 2015, Version 2. Eur Urol. 2016; 69:16-40

18. Edge SB, Compton CC. The American Joint Committee on Cancer: the 7th edition of the AJCC cancer staging manual and the future of TNM. Ann Surg Oncol. 2010; 17:1471-4.

19. D'Amico AV, Whittington R, Malkowicz SB, et al. Biochemical outcome after radical prostatectomy, external beam radiation therapy, or interstitial radiation therapy for clinically localized prostate cancer. JAMA. 1998; 280:969-74.

20. Porcaro AB, De Luyk N, Corsi P, et al. Clinical factors predicting bilateral lymph node invasion in high-risk prostate cancer. Urol Intern. 2017; 99:392-9.

21. Mottet N, Bellmunt J, Bolla $M$, et al. EAU-ESTRO-SIOG Guidelines on Prostate Cancer. Part 1: Screening, Diagnosis, and Local Treatment with Curative Intent. Eur Urol. 2017; 71:618-29.

22. Briganti A, Larcher A, Abdollah F, et al. Updated nomogram predicting lymph node invasion in patients with prostate cancer undergoing extended pelvic lymph node dissection: the essential importance of percentage of positive cores. Eur Urol. 2012; 61:480-7.

23. Grasso AA, Cozzi G, E. Del, et al. Multicenter analysis of pathological outcomes of patients eligible for active surveillance according to PRIAS criteria. Minerva Urol Nefrol. 2016; 68:237-41.

24. Menon M, Tewari A, Peabody J. Vattikuti Institute prostatectomy: technique. J Urol. 2003; 169:2289-92.

25. Porreca A, Salvaggio A, Dandrea M, et al. Robotic-assisted radical prostatectomy with the use of barbed sutures. Surg Technol Intern. 2017; 30:39-43

26. Bianchi FM, Romagnoli D, D'Agostino D, et al. Posterior muscle-fascial reconstruction and knotless urethro-neo bladder anastomosis during robot-assisted radical cystectomy: Description of the technique and its impact on urinary continence Arch Ital Urol Androl. 2019: 91:5-10.

27. Vagnoni V, Brunocilla E, Bianchi L, et al. State of the art of PET/CT with 11-choline and 18F-fluorocholine in the diagnosis and follow-up of localized and locally advanced prostate cancer. Arch Esp Urol. 2015; 68:354-70

28. Gacci M, Noale M, Artibani W, et al. Quality of Life After Prostate Cancer Diagnosis: Data from the Pros-IT CNR. Eur Urol Focus. 2017; 3:321-324.

29. D'Agostino D, Bianchi FM, Romagnoli D, et al. MRI/TRUS FUSION guided biopsy as first approach in ambulatory setting:
Feasibility and performance of a new fusion device. Arch Ital Urol Androl. 2020; 91:211-217.

30. D'Agostino D, Bianchi FM, Romagnoli D, et al. Comparison between "In-bore" MRI guided prostate biopsy and standard ultrasound guided biopsy in the patient with suspicious prostate cancer: Preliminary results. Arch Ital Urol Androl. 2019; 91:87-92.

31. Porreca A, D'Agostino D, Vigo M., et al. "in-bore" MRI prostate biopsy is a safe preoperative clinical tool to exclude significant prostate cancer in symptomatic patients with benign prostatic obstruction before transurethral laser enucleation Arch Ital Urol Androl. 2020; 91:224-229.

32. Villers A, Puech P, Flamand V, et al. Partial prostatectomy for anterior Cancer: short-term oncologic and functional outcomes. Eur Urol. 2017; 72:333-342.

33. Koppie TM, Bianco FJ Jr, Kuroiwa K, et al. The clinical features of anterior prostate cancers. BJU Int. 2006; 98:1167-1171.

34. Takahashi H, Epstein JI, Wakui S, et al. Difference in prostate cancer grade, stage, and ocation in radical prostatectomy specimens from United States and Japan. Prostate 2014; 74:321-5.

35. Volkin D, Turkbey B, Hoang AN, et al. Multiparametric magnetic resonance imaging (MRI) and subsequent MRI/ultrasonographyfusion-guided biopsy increase the detection of anteriorly located prostate cancers. BJU Int. 2014; 114:E43-E49.

36. Hossac T, Patel MI, Huo A, et al. Location and pathological characteristics of cancers in radical prostatectomy specimens identified by transperineal biopsy compared to transrectal biopsy. J Urol. 2012; 188:781-5.

37. Mygatt J, Sesterhenn I, Ronser I, et al. Anterior tumors of the prostate: Clinicopathological features and outcomes. Prostate Cancer Prostatic Dis. 2014; 17:75-80

38. Al-Ahmadie HA, Tickoo SK, Olgac S, et al. Anterior-predominant prostatic tumors: zone of origin and pathologic outcomes at radical prostatectomy. Am J Surg Pathol. 2008; 32:229-35.

39. Hossac T, Patel MI, Huo A, et al. Location and pathological characteristics of cancers in radical prostatectomy specimens identified by transperineal biopsy compared to transrectal biopsy. J Urol. 2012; 188:781-5.

\section{Correspondence}

Daniele D’Agostino, MD (Corresponding Author) dott.dagostino@gmail.com

Paolo Corsi, MD - pcorsi@casacura.it

Michele Colicchia, MD - mcolicchia@casacura.it

Daniele Romagnoli, MD - dromagnoli@casacura.it

Angelo Porreca, MD

Department of Urology, Abano Terme Hospital

Piazza Cristoforo Colombo 1, 35031, Abano Terme (PD) (Italy)

Gian Maria Busetto, MD - gianmaria.busetto@uniromal.it

Department of Urology, Università "la Sapienza", Roma

Matteo Ferro, MD - matteo.ferro@ieo.it

European Institute of Oncology, Milan

Alessandro Tafuri, MD - aletaf@hotmail.it

Matteo Cevenini, MD - matteoceve@gmail.com

Federico Mineo Bianchi, MD - federico.mineobianchi@gmail.com

Department of Urology, University of Verona

Marco Giampaoli, MD - mgiampaoli@casacura.it

Department of Urology, University of Bologna 